\section{Headache associated with presence of IUS}

A 19-year-old woman had a Mirena ${ }^{\circledR}$ intrauterine system (IUS) fitted for contraception 10 weeks postnatally. She attended our clinic 4 weeks later and reported apparent migraine with aura, which had developed within a few days of the fitting. Initially, she tried paracetamol and ibuprofen, which were not helpful, and saw her general practitioner who prescribed a triptan. The headaches were becoming increasingly severe, and she requested removal of the IUS.

She had no pelvic pain and no vaginal bleeding apart from slight spotting after the IUS insertion. She was a non-smoker with no personal risk factors for vascular disease and no family history of vascular disease. She was not taking any prescribed or over-the-counter medication or supplements.

The patient had experienced migraine with aura as an adolescent but had not had any for 5 years, and had never had such severe symptoms. Before her pregnancy she had used a progestogen-only pill (Cerelle ${ }^{\circledR}$ ) and Nexplanon ${ }^{\circledR}$ implant without experiencing any headaches or symptoms of migraine.

When a detailed history of her headaches was taken, she described the visual symptoms as being 'sparkles' in front of her eyes for a few minutes at a time, and black spots in her vision which made her feel as if she was going to faint. These symptoms did not precede the headaches but occurred at the same time. The headaches were sometimes unilateral, and sometimes frontal with associated nausea but no vomiting. They lasted for several hours, and in the few days before her clinic appointment had been waking her at night and were present all the time.

The IUS was removed at her clinic visit, and when she was reviewed 1 week later she reported that her symptoms had resolved completely within 10 hours of removal of the IUS. In a further telephone consultation 3 months later she told me that she started Cerelle 2 weeks after the IUS removal and was well with no further headaches.

This case raises interesting questions about the cause of these headaches. Was this due to the levonorgestrel (LNG) or was it due to the presence of an intrauterine device (IUD)? She reported that her symptoms had completely disappeared within 10 hours of IUS removal, but the half-life of LNG is 20 (range 980 ) hours, and she had had no previous problems with the known higher serum levels of desogestrel with Cerelle and Nexplanon. It would be interesting to see if she had a similar response to a copper IUD, but unsurprisingly she does not wish to try intrauterine contraception again. This case also illustrates the diagnostic difficulties of migraine with aura, as this did not appear to be a true aura, and her visual symptoms associated with feeling faint could have been a vasovagal response.

In a 2013 review article on contraception and headache, no studies were identified that assessed the effect of the IUS on migraine, but there were several randomised controlled trials showing increased reporting of headaches in IUS users compared to those with copper IUDs. ${ }^{1}$ There has been a similarly dramatic case report of trigeminal autonomic cephalgia following IUS insertion published in the letters page of this Journal. ${ }^{2}$ I would be interested to know if any Journal readers have had similar cases.

Caroline Cooper, MRCGP, MFSRH

Associate Specialist, Sexual and Reproductive Health - Cambridge iCASH, Lime Tree Clinic, Cambridge, UK; caroline.cooper@nhs.net

Competing interests The author has received honoraria from Bayer Pharma for lecturing and training healthcare professionals to fit IUD/IUS.

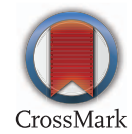

Published Online First 14 October 2015

J Fam Plann Reprod Health Care 2016;42:75. doi:10.1136/fprhc-2015-101309

\section{REFERENCES}

1 MacGregor A. Contraception and headache. Headache 2013;53:247-276.

2 Lipscombe S. IUS producing a TAC. J Fam Plann Reprod Health Care 2009;35:132. 